\title{
Commentary: The coronary Gordian knot
}

\author{
Kenza Rahmouni, MDCM, and \\ Fraser D. Rubens, MD, MSc, FACS, FRCSC
}

Scoring systems serve a common goal to standardize the classification of complex conditions to improve decisionmaking. When properly built, they can predict outcomes and assist in patient stratification for clinical trials. In the field of coronary artery disease (CAD), the SYNTAX (Synergy Between Percutaneous Coronary Intervention with TAXUS and Cardiac Surgery) score has become widely used to classify the severity of coronary lesions, and it has been integrated into a culture that guides the choice of revascularization strategy between percutaneous coronary intervention (PCI) and coronary artery bypass grafting (CABG).

When first introduced, the SYNTAX score was never intended for use as a predictive tool but rather to objectively quantify the severity of CAD in enrolled patients. ${ }^{1}$ It was only post-hoc analyses of the SYNTAX cohort that demonstrated the score's ability to predict major adverse cardiac and cerebrovascular events in patients undergoing PCI. ${ }^{1-3}$ Although surgery was overall superior in terms of major adverse cardiac and cerebrovascular events, there was no apparent benefit of CABG versus PCI in the low (SYNTAX score $\leq 22$ ) and intermediate (SYNTAX score $>22$ and $\leq 33$ ) tertiles. ${ }^{4}$

These findings have perpetuated a narrative that favors PCI in low SYNTAX scores and CABG in high SYNTAX scores, even though in the original SYNTAX trial, there was no relationship between surgical outcomes and CAD complexity and in fact risk decreased with increasing

\footnotetext{
From the Division of Cardiac Surgery, University of Ottawa Heart Institute, Ottawa, Ontario, Canada.

Disclosures: The authors reported no conflicts of interest.

The Journal policy requires editors and reviewers to disclose conflicts of interest and to decline handling or reviewing manuscripts for which they may have a conflict of interest. The editors and reviewers of this article have no conflicts of interest.

Received for publication June 1, 2021; revisions received June 1, 2021; accepted for publication June 1, 2021; available ahead of print June 19, 2021.

Address for reprints: Fraser D. Rubens, MD, MSc, FACS, FRCSC, University of Ottawa Heart Institute, 40 Ruskin St, Ottawa, Ontario, Canada K1Y 4W7 (E-mail: FRubens@ottawaheart.ca).

J Thorac Cardiovasc Surg 2023;165:1414

0022-5223/ $\$ 36.00$

Copyright (c) 2021 by The American Association for Thoracic Surgery

https://doi.org/10.1016/j.jtcvs.2021.06.002
}

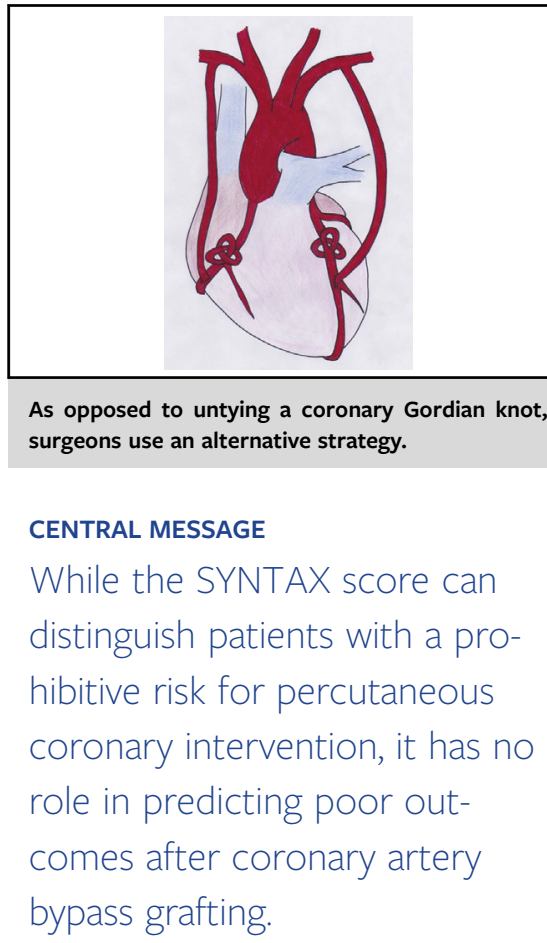

scores. Subsequent trials comparing $\mathrm{CABG}$ and PCI have further supported that surgical outcomes are poorly correlated with SYNTAX score and that prognosis is better related to patient clinical profile and procedural variables.

In this meta-analysis comparing PCI and CABG, Gaudino and colleagues ${ }^{5}$ hypothesized that the benefit of CABG versus PCI would not vary substantially according to SYNTAX scores. To prove their hypothesis, the authors performed a sensitivity analysis using ratios of incidence rate ratios to generate interaction effect estimates and compare outcomes between each SYNTAX score tertile. When combining the results from SYNTAX and other large trials, (including FREEDOM [Future Revascularization Evaluation in Patients with Diabetes Mellitus: Optimal Management of Multivessel Disease] and NOBLE [NordicBaltic-British Left Main Revascularization]), they found that the incidence rate ratio was significantly in favor of CABG in low, intermediate, and high SYNTAX score groups (1.25 [1.02-1.54], 1.48 [1.30-1.69], and 1.39 [1.181.63], respectively). This emphasizes that the narrative of PCI being indicated in the lower tertiles is fallacious, since surgery is superior in all tertiles. SYNTAX probably only indicated to us that PCI risk in the highest tertile was arguably prohibitive. 
These results are not surprising, since we know that the SYNTAX score (a surrogate for coronary disease complexity) lies within the causal pathway for PCI and late outcomes, and behaves as a mediator of outcomes. ${ }^{2,5}$ In contrast, there is no direct relationship between the SYNTAX score and outcomes after CABG because this mediating effect is absent. ${ }^{2}$ In fact, the complexity of the coronary artery lesions has little effect on the quality of the revascularization because surgeons bypass the diseased portions of the vessel. ${ }^{2}$ Surgeons approach the conundrum of coronary insufficiency not by unravelling the knot but by distal bypass rendering coronary complexity moot in the same manner as Alexander the Great handled the Gordian Knot.

\section{References}

1. Serruys PW, Hara H, Onuma Y. Did the SYNTAX score pass the test of time? JACC Cardiovasc Interv. 2020;13:1207-10.

2. Yadav M, Palmerini T, Caixeta A, Madhavan MV, Sanidas E, Kirtane AJ, et al. Prediction of coronary risk by SYNTAX and derived scores: synergy between percutaneous coronary intervention with taxus and cardiac surgery. J Am Coll Cardiol. 2013;62:1219-30.

3. ClinicalTrials.gov. SYNTAX study: TAXUS drug-eluting stent versus coronary artery bypass surgery for the treatment of narrowed arteries (SYNTAX) 2005. Accessed June 6, 2021. Available at: https://clinicaltrials.gov/ct2/show/ NCT00114972?term $=$ NCT00114972 \&draw $=2 \&$ rank $=1$

4. Serruys PW, Morice MC, Kappetein AP, Colombo A, Holmes DR, Mack MJ, et al Percutaneous coronary intervention versus coronary-artery bypass grafting for severe coronary artery disease. $N$ Engl J Med. 2009;360:961-72.

5. Gaudino M, Hameed I, Di Franco A, Naik A, Demetres M, Biondi-Zoccai G, et al Comparison of SYNTAX score strata effects of percutaneous and surgical revascularization trials: a meta-analysis. J Thorac Cardiovasc Surg. 2023;165: 1405-13.e13.
See Article page 1405 .

\section{Commentary: Does the SYNTAX (Synergy between PCI with Taxus and Cardiac Surgery) score even matter?}

\author{
Derrick Y. Tam, MD, PhD, ${ }^{\text {a }}$ Marc Ruel, MD, MPH, ${ }^{\mathrm{b}}$ \\ and Stephen E. Fremes, MD, MSc ${ }^{\mathrm{a}}$
}

The advent of revascularization trials comparing drugeluting stents (DES) with coronary artery bypass grafting $(\mathrm{CABG})$ prompted the need to more accurately quantify the complexity of coronary artery disease (CAD). In the design of the Synergy between PCI with Taxus and Cardiac Surgery (SYNTAX) trial of DES versus CABG for multivessel $C A D$, the realization that $C A D$ severity measured by the number of diseased epicardial vessels alone was

\footnotetext{
From the a Division of Cardiac Surgery, Schulich Heart Centre, Department of Surgery, Sunnybrook Health Sciences Centre, University of Toronto, Toronto; and ${ }^{\mathrm{b}} \mathrm{Di}$ vision of Cardiac Surgery, University of Ottawa Heart Institute, Ottawa, Ontario, Canada.

Disclosures: The authors reported no conflicts of interest.

The Journal policy requires editors and reviewers to disclose conflicts of interest and to decline handling or reviewing manuscripts for which they may have a conflict of interest. The editors and reviewers of this article have no conflicts of interest.

Received for publication June 17, 2021; revisions received June 17, 2021; accepted for publication June 18, 2021; available ahead of print June 26, 2021.

Address for reprints: Derrick Y. Tam, MD, PhD, Division of Cardiac Surgery, University of Toronto, 200 Elizabeth St, Toronto, Ontario M5G 2C4, Canada (E-mail: derricky.tam@gmail.com).

J Thorac Cardiovasc Surg 2023;165:1415-7

$0022-5223 / \$ 36.00$

Copyright (C) 2021 by The American Association for Thoracic Surgery

https://doi.org/10.1016/j.jtcvs.2021.06.037
}

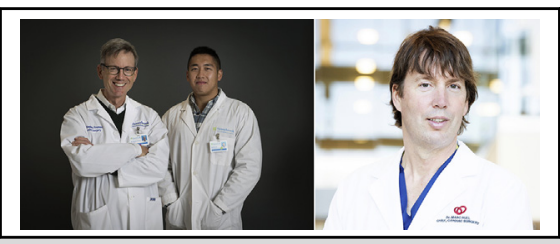

Stephen E. Fremes, MD, MSc, Derrick Y. Tam, MD, $\mathrm{PhD}$, and Marc Ruel, MD, MPH

\begin{abstract}
CENTRAL MESSAGE
In patients with multivessel disease, coronary artery bypass grafting is associated with improved outcomes compared with drug-eluting stents regardless of anatomical complexity.
\end{abstract}

not sufficient gave way to the development of the SYNTAX score. ${ }^{1}$ The SYNTAX score incorporates several important factors to quantify the severity and complexity of CAD, some of which include lesion location, vessel caliber and length, degree of stenosis, the amount of myocardium at risk, and degree of calcification. Retrospective early validation was performed in 306 patients from the percutaneous coronary intervention (PCI) arm of the Arterial Revascularization Therapies Study part II (ARTS-II) trial, which 